EESTI NSV TEADUSTE AKADEEMIA TOIMETISED. 29. KOIDË FOUSIKA * MATEMAATIKA. 1980, NR. 4

ИЗВЕСТИЯ АКАДЕМИИ НАУК ЭСТОНСКОП ССР. ТОМ 29 ФИЗИКА * МАТЕМАТИКА, 1980, № 4

\title{
ПОЛЯРОН В ТОНКОЙ ДИЭЛЕКТРИЧЕСКОЙ ПЛЕНКЕ
}

\author{
(Представил В. Хижняков)
}

Исследования элементарных возбуждений поверхностей кристалла и тонких кристаллических пленок приобрели в последнее время актуальность в связи с развитием техники. В первую очередь это относится к фононным и электронным элементарным возбуждениям (см. $\left.\left[{ }^{1}\right]\right)$. В $\left.{ }^{2}\right]$ было показано, что в полупроводниковой пленке, диэлектрическая проницаемость которой значительно превышает единицу, происходит существенное усиление кулоновского взаимодействия по сравнению с бесконечным кристаллом, когда толщина пленки становится порядка или меньше расстояния между зарядами. Этот эффект приводит к усилению взаимодействия электяона проводимости с поляризацией, вызванной оптическими колебаниями пленки полярного кристалла, толщина которой приближается к радиусу полярона. При этом могут возрастать энергия связи полярона и его масса, что будет зарегистрировано оптическими измерениями нли измерениями подвижности носителей. Однако отнюдь не очевидно, произойдет ли в действительности указанное возрастание, поскольку в случае приближения толщины пленки к радиусу полярона происходит его деформация, что энергетически невыгодно. Данная работа посвящена исследованию этого вопpoca.

Используется модель полярона, предложенная С. И. Пекаром [3]. Эта модель пригодна в случае сильной связи и большого характерного размера полярона $L$ :

$$
\left|E_{b}\right| \gg \hbar_{\omega_{0}}, \quad L \gg a .
$$

Здесь $E_{b}<0$ - энергия покоящегося полярона, отсчитанная от дна зоны проводимости, $\omega_{0}-$ частота $L O$-фонона, $a$ - постоянная решетки. Благодаря условиям (1) пленку можно рассматривать как диэлектрический континуум и использовать адиабатическое приближение. Гамильтониан электрон-фононной системы пленки в этом случае имеет вид

$$
\begin{gathered}
H=\frac{p^{2}}{2 m}+U(z)-e \sum_{i} \int d^{3} r^{\prime} P_{i}\left(\vec{r}^{\prime}\right) \frac{\partial}{\partial r_{i}^{\prime}} V\left(\vec{r}, \overrightarrow{r^{\prime}}\right)+\frac{2 \pi}{\varepsilon_{0}-\varepsilon} \int d^{3} r^{\prime} P^{2}\left(\overrightarrow{r^{\prime}}\right)+ \\
+\frac{1}{2} \sum_{i, j} \iint d^{3} r^{\prime} d^{3} r^{\prime \prime} P_{i}\left(\vec{r}^{\prime}\right) P_{j}\left(\vec{r}^{\prime \prime}\right) \frac{\partial^{2}}{\partial r_{i}^{\prime} \partial r_{j}^{\prime \prime}} V\left(\overrightarrow{r^{\prime}}, \vec{r}^{\prime \prime}\right) .
\end{gathered}
$$

Здесь $\vec{p}$ - импульс электрона, $m$ - его эффективная масса, $P_{i}-$ компоненты вектора поляризации, которые обусловлены лишь смеще- 
ниями ионов (в отличие от $\left[{ }^{3}\right]$ здесь в $\vec{P}$ не включается вызванная этими смещениями электронная поляризация), $\varepsilon_{0}$ и $\varepsilon-$ статическая и высокочастотная диэлектрические проницаемости соответственно, $V\left(\vec{r}, \overrightarrow{r^{\prime}}\right)$ - потенциал, создающийся в пленке с диэлектрической проницаемостью $\varepsilon$ в точке $\vec{r}$ единичным точечным зарядом, находящимся в точке $\overrightarrow{r^{\prime}}$ (для простоты считаем, что пленка помещена в вакуум). Вид этого потенциала можно найти, используя стандартную методику $\left[{ }^{4}\right]$ :

$$
\begin{gathered}
V\left(\vec{r}, \overrightarrow{r^{\prime}}\right)=(2 \pi \varepsilon)^{-1} \int d^{2} k f\left(k, z, z^{\prime}\right) \exp \left[\overrightarrow{i k}\left(\overrightarrow{\varrho^{\prime}}-\vec{\varrho}\right)\right] / k, \\
f\left(k, z, z^{\prime}\right)=\exp \left[-k\left|z-z^{\prime}\right|\right]-\left\{2 \operatorname{ch}\left[k\left(z-z^{\prime}\right)\right]+x \exp \left[k\left(z^{\prime}+z+2 d\right)\right]+\right. \\
\left.+x \exp \left[-k\left(z+z^{\prime}\right)\right]\right\} /\left[1-x^{2} \exp (2 k d)\right],
\end{gathered}
$$

где $\vec{k}$ - двумерный вектор, параллельный поверхностям пленки, $\overrightarrow{\mathrm{e}}$ и $\overrightarrow{\mathrm{e}^{\prime}}$ также двумерные векторы, возникающие при проектировании векторов $\vec{r}$ и $\overrightarrow{r^{\prime}}$ на указанные поверхности. Система координат выбрана таким образом, что эти поверхности перпендикулярны оси $\vec{z}$ и пересекают ее в точках 0 и $-d$. $x=(\varepsilon+1) /(\varepsilon-1)$. При $\left|\overrightarrow{\varrho^{\prime}}-\vec{\varrho}\right| \gg d$ основной вклад в интеграл дают малые $k: k d \ll 1$, и формула (3) упрощается (см. также $\left.\left[{ }^{2}\right]\right)$ :

$$
V\left(\vec{r}, \overrightarrow{r^{\prime}}\right) \approx(\pi \varepsilon d)^{-1} \int d^{2} k \exp \left[\overrightarrow{i k}\left(\overrightarrow{\varrho^{\prime}}-\overrightarrow{\mathrm{Q}}\right)\right] /[k(k+2 /(\varepsilon d))] .
$$

Предполагается, что $\varepsilon \gg 1$. Дно зоны проводимости, как правило, лежит по энергии на несколько электрон-вольт ниже уровня вакуума [5]. Эта величина значительно превышает расстояние между размерноквантованными уровнями энергии $\hbar^{2} \pi^{2} /\left(2 m d^{2}\right)$ для рассматриваемых здесь пленок. Аппроксимируем существующие вблизи поверхностей пленки энергетические барьеры ступенчатыми функциями, причем в силу последнего обстоятельства будем считать, что образующаяся потенциальная яма имеет бесконечно высокие стенки: $U(z)=0$ при $-d<z<0 ; \quad U(z)=\infty$ при $z \leqslant-d$ и $z \geqslant 0$. Такое приближение оправдано для двумерных подзон, образующихся из нескольких нижайших размерно-квантованных уровней.

Третье слагаемое в правой части (2) описывает взаимодействие поляризации с электроном, четвертое - упругую энергию, связанную с поляризацией пленки, плюс слагаемое, обусловленное полем Лоренца $\left[{ }^{6}\right]$. Наконец, пятый член в правой части формулы (2) есть кулоновская энергия поляризации минус отмеченное выше слагаемое, вызванное полем Лоренца. Взаимодействие электрона с вызванной им безынерционной поляризацией смещений электронных оболочек ионов включено, как и в $\left[{ }^{3}\right]$, в определение эффективной массы электрона. Для рассматриваемых здесь макроскопически толстых пленок $(d \gg a)$ последняя величина слабо зависит от толщины пленки, поскольку радиус безынерционной поляризации микроскопически мал $\left[{ }^{7}\right]$.

Гамильтониан (2) можно привести к виду, использованному в $\left[{ }^{3}\right]$, если устремить $d$ к бесконечности в (3) и учесть, что для бесконечной однородной изотропной среды

$$
\vec{D}(\vec{r})-\vec{E}(\vec{r})=\vec{E}^{\prime}(\vec{r})-\vec{E}(\vec{r})=
$$




$$
=-\int d^{3} r^{\prime}\left[3 \vec{R} \cdot \vec{P}\left(\vec{r}^{\prime}\right) \vec{R}-\vec{P}\left(r^{\prime}\right) R^{2}\right] / R^{5}=4 \pi \vec{P}(\vec{r})
$$

и $\vec{P}(\vec{r})=\varepsilon \overrightarrow{P^{\prime}}(\vec{r})$. Здесь $\vec{D}(\vec{r})$ - вектор электрической индукции, $\vec{E}^{\prime}(\vec{r})$-напряженность поля свободных зарядов, $\quad \vec{R}=\vec{r}-\overrightarrow{r^{\prime}}, \overrightarrow{P^{\prime}}(\vec{r})-$ поляризация, созданная смещением ионов и связанной с этим смещением деформацией их электронных оболочек. Во вторично-квантованном виде гамильтониан (2) совпадает с полученными ранее другими методами $\left[{ }^{7,8}\right]$.

Как и в $\left[{ }^{3}\right]$, используем для нахождения энергии покоящегося полярона прямой вариационный метод. Пленка предполагается достаточно тонкой, так что характерная энергия размерно-квантованных уровней $\hbar^{2} \pi^{2} /\left(2 m d^{2}\right)$ значительно превышает величину кулоновского взаимодействия между электроном и поляризацией (см. ниже). Поэтому поперечное относительно пленки движение электрона не меняется при учете взаимодействия с фононами и его волновую функцию следует искать в виде $\psi \overrightarrow{(r)}=\psi(\vec{\varrho}) \psi(z), \quad$ где $\psi(z)$ - одна из волновых функций частицы, локализованной в прямоугольной яме с бесконечно высокими стенками. Функционал, соответствующий гамильтониану (2), имеет вид

$$
\begin{gathered}
F=-\hbar^{2}(2 m)^{-1} \int d^{2} \varrho \psi^{*}(\vec{\varrho}) \underset{\rho}{\Delta \rightarrow} \underset{\rho}{(\varrho)}-\int d^{3} r \vec{P}(\vec{r}) \cdot \vec{E}(\vec{r})+ \\
+2 \pi\left(\varepsilon_{0}-\varepsilon\right)^{-1} \int d^{3} r P^{2}(\vec{r})+ \\
+(1 / 2) \sum_{i, j} \iint d^{3} r^{\prime} d^{3} r^{\prime \prime} P_{i}\left(\vec{r}^{\prime}\right) P_{j}\left(\vec{r}^{\prime \prime}\right) \frac{\partial^{2}}{\partial r_{i}^{\prime} \partial r_{j}^{\prime \prime}} V\left(\overrightarrow{r^{\prime}}, \vec{r}^{\prime \prime}\right), \\
\vec{E}(\vec{r})=e \operatorname{grad} \int d^{3} r^{\prime}\left|\psi\left(\overrightarrow{r^{\prime}}\right)\right|^{2} V\left(\vec{r}, \vec{r}^{\prime}\right),
\end{gathered}
$$

где $\underset{\vec{p}}{\Delta_{\rightarrow}}=\partial^{2} / \partial x^{2}+\partial^{2} / \partial y^{2}$, энергия отсчитывается от дна соответствующей подзоны. Варьируя $F$ вначале по $\vec{P}(\vec{r})$, находим необходимое условие минимума

$$
-E_{i}(\vec{r})+\frac{\partial}{\partial r_{i}} \sum_{j} \int d^{3} r^{\prime} P_{j}\left(\overrightarrow{r^{\prime}}\right) \frac{\partial}{\partial r_{j}^{\prime}} V\left(\vec{r}, \vec{r}^{\prime}\right)+4 \pi\left(\varepsilon_{0}-\varepsilon\right)^{-1} P_{i}(\vec{r})=0
$$

при выполнении которого (5) принимает вид

$$
F=-\hbar^{2}(2 m)^{-1} \int d^{2} \varrho \psi^{*}(\vec{\varrho}) \underset{\rho}{\Delta \rightarrow} \underset{\rho}{(\varrho)}-(1 / 2) \int d^{3} r \vec{P}(\vec{r}) \cdot \vec{E}(\vec{r}) .
$$

Для дальнейших вычислений необходимо, исходя из (7), явно выразить $\vec{P}(\vec{r})$ через $\psi(\vec{r})$. Для этого подействуем на $(7)$ оператором $\sum_{i} \partial / \partial r_{i}$. Учитывая, что $\underset{r}{\Delta_{r}} V\left(\vec{r}, \overrightarrow{r^{\prime}}\right)=-4 \pi \delta\left(\vec{r}-\overrightarrow{r^{\prime}}\right) / \varepsilon, \quad$ находим

$$
4 \pi e|\psi(\vec{r})|^{2} / \varepsilon_{0}+\operatorname{div} \vec{E}_{0}(\vec{r})=0,
$$

где $\vec{E}_{0}(\vec{r})=4 \pi \vec{P}(\vec{r}) /\left(\varepsilon_{0}-\varepsilon\right)$ - вектор электрического поля в пленке. Уравнения и граничные условия, которым подчиняются полное электри- 
ческое поле $\vec{E}_{0}(\vec{r})$ и поле электрона $\vec{E}(\vec{r})$ (6), совпадают с точностью до замены $\varepsilon_{0}$ на $\varepsilon$. Таким образом, решение уравнения (9) может быть записано сразу:

$$
\vec{P}(\vec{r})=(4 \pi)^{-1} e\left(\varepsilon_{0}-\varepsilon\right) \operatorname{grad} \int d^{3} r^{\prime}\left|\psi\left(\overrightarrow{r^{\prime}}\right)\right|^{2} V_{0}\left(\vec{r}, \overrightarrow{r^{\prime}}\right),
$$

где $V_{0}\left(\vec{r}, \overrightarrow{r^{\prime}}\right)$ определяется формулами (3) и (4) с заменой $\varepsilon$ на $\varepsilon_{0}$. В том, что решение (10) удовлетворяет уравнению (7), легко убедиться, используя граничные условия и теорему Гаусса-Остроградского.

В качестве аппроксимирующей функции выберем

$$
\psi(\varrho)=\alpha \sqrt{2 / \pi} \exp (-\alpha \varrho), \quad \int d^{2} \varrho \psi^{2}(\varrho)=1 .
$$

Предположим для простоты, что $2 \alpha^{-1} \gg d$. В этом случае для $V\left(\vec{r}, \overrightarrow{r^{\prime}}\right)$ и $V_{0}\left(\vec{r}, \overrightarrow{r^{\prime}}\right)$ можно использовать приближенную формулу (4). Отметим, что в таком приближении энергии связи поляронов, образо-ванных различными подзонами, одинаковы, поскольку $V\left(\vec{r}, \overrightarrow{r^{\prime}}\right)$ в формуле (4) не зависит от $z$ и $z^{\prime}$. Подставляя (11) в выражения (6) и (10), находим из (8)

$$
\begin{gathered}
F=\left(2 m d^{2} y^{2}\right)^{-1}+\left[C(y)-C_{0}(y)\right] e^{2} / d, \\
C(y)=\varepsilon^{-1}(y / \varepsilon)\left(y^{2} / \varepsilon^{2}+1\right)^{-3}\left\{\pi\left[3\left(\varepsilon^{2} / y^{2}-2\right)-y^{2} / \varepsilon^{2}\right] / 16+\right. \\
\left.+\left[1+y^{2} /\left(4 \varepsilon^{2}\right)\right] y / \varepsilon+[3 / 4+\ln (y / \varepsilon)] \varepsilon / y\right\},
\end{gathered}
$$

где $y=\alpha^{-1} / d, C_{0}(y)$ выражается той же формулой, что и $C(y)$ с заменой $\varepsilon$ на $\varepsilon_{0}$.

Выберем для примера следующие значения параметров: $\varepsilon=3$, $\varepsilon_{0}=15, m=0,1 m_{e}\left(m_{e}-\right.$ масса свободного электрона), $d=20 \AA$. Минимизируя $F$, находим: $F_{\min }=E_{b}=-0,237 e^{2} /(€ d) \approx-50$ мэB , $y_{\min }=1,6\left(2 \alpha_{\min }^{-1} / d=3,2 \gg 1\right), \epsilon=\varepsilon_{0} \varepsilon /\left(\varepsilon_{0}-\varepsilon\right)$. Это значение более чем в четыре раза превышает энергию связи полярона в бесконечном кристалле в модели сильной связи $E_{P}=-0,0544 m e^{4} /(\hbar \epsilon)^{2} \quad\left[{ }^{3}\right]$. Полученная в этой последней модели поляронная яма, однако, слишком мелка. Сравним поэтому $E_{b}$ с результатом, полученным в модели промежуточ,ной и слабой связи $E_{F}=-e^{2} \sqrt{2 m \omega_{0} / \hbar} /(2 €) \quad\left[{ }^{9}\right]$. При $\omega_{0}=1,5 \cdot 10^{13} c^{-1}$ найденное значение $E_{b}$ примерно в полтора раза превышает $E_{F}$. Заметим, что для принятой величины $\omega_{0}$ выполняется первое условие (1), а также, что для приведенных значений параметров энергия кулоновского взаимодействия электрона и поляризации $\int d^{3} r \vec{P} \cdot \vec{E} \approx e^{2} /(€ d)$

значительно меньше расстояния между размерно-квантованными уровнями. Поэтому поперечное относительно пленки движение электрона, как и предполагалось, не изменяется при учете взаимодействия с поляризацией.

Таким образом, в тонкой пленке полярного диэлектрика происходит возрастание энергии связи (а следовательно, и массы) полярона. В связи с этим отметим работу $\left[{ }^{10}\right]$, результатом которой являлось утверждение, что энергия связи полярона (вычислявшаяся в модели слабой связи) монотонно убывает с уменьшением толщины пленки. По-видимому, этот результат обусловлен тем, что в $\left[{ }^{10}\right]$ не принималось во внимание взанмодействие с поверхностными фононами, которое стано- 
вится существенным именно для тонких пленок. Использованная малая толщина пленки $(d=20 \AA)$ «навязана» условием $2 \alpha^{-1} \gg d$, позволившим значительно упростить математические выражения. По-видимому, отмеченный-эффект может наблюдаться и в слоях, на порядок более толстых.

Автор признателен В. В. Хижнякову за обсуждение результатов работы.

\section{ЛИТЕРАТ У РА}

1. Брыксин В. В., Мирлин Д. Н., Фирсов Ю. А., Успехи физ. наук, 113, вып, 1, 29-67 (1974).

2. Келдыш Л. В., Письма в ЖЭТФ, 29, вып. 11, 716-719 (1979).

3. Пек ар С. И., Исследования по электронной теории кристалов, М.-Л., ГИТТЛ, 1951; Успехи физ. наук, 50, вып. 2, 197-252 (1953).

4. С м а й т В., Электростатика и электродинамика, М., ИИЛ, 1954.

5. Р и в ере Х., В кн.: Поверхностные свойства твердых тел, М., «Мир», 1972, c. $193-316$.

6. Т а м м И. Е., Основы теории электричества, М., «Наука», 1976.

7. L i c ari, J. J., Evrard, R., Phys. Rev., B15, № 4, 2254-2264 (1977).

8. Брыксин В. В., Фирсов Ю. А.. Физ. твердого тела, 13, вып. 2, 496-503 (1971); Lucas, A. A., Kartheuser, E., B adro, R. G., Phys. Rev., B2, № 7, 2488-2499 (1970).

9. Lee, T. D., Low, F. E., Pines, D., Phys. Rev., 90, № 2, 297-302 (1953).

10. L i c a ri, J. J., Solid State Commun., 29, № 8, 625-628 (1979).

$\begin{array}{cc}\text { Институт физики } & \text { Поступила в редакцию } \\ \text { Академии наук Эстонской ССP } & \text { 9/VI } 1980\end{array}$

A. SERMAN.

\section{POLARON OHUKESES DIELEKTRILISES KILES}

On arvutatud tugevasti seotud kontinuaalpolaroni omaenergia suure kõrgsagedusliku dielektrilise läbitavusega kiles, mille paksus on polaroni raadiuse suurusjärgus. On näidatud, et see omaenergia võib olla tunduvalt suurem polaroni seoseenergiast lōpmatus kristallis.

\section{A. SERMAN}

\section{THE POLARON IN A THIN DIELECTRIC FILM}

A Hamiltonian is obtained and a self-energy of a strong-coupling continual polaron is investigated for a thin dielectric film placed in vacuum. As it was shown by L. V. Keldysh $\left[{ }^{2}\right]$, the Coulomb interaction increases in the film surrounded by the environment with a smaller dielectric constant in case the thickness of the film tends to a distance between charges. This effect may lead to the growth of the polaron self-energy and mass in a film with a large high-frequency dielectric constant as its thickness becomes of the order of the polaron radius. This growth is prevented by a simultaneously arising polaron deformation. The calculations of the self-energy are carried out by the straight variational method. It is shown that its value may really considerably exceed the coupling energy of the polaron in an infinite crystal. 